\title{
Potenciais medicinais da faveleira (Cnidoscolus quercifolius) e seus usos na saúde humana: uma breve revisão
}

Medicinal potentials of the faveleira (Cnidoscolus quercifolius) and its uses in human health: a brief review

\section{Potencialidades medicinales de la feveleira (Cnidoscolus quercifolius) y sus usos en la salud}

humana: una breve revisión

Recebido: 10/02/2021 | Revisado: 14/02/2021 | Aceito: 14/02/2021 | Publicado: 22/02/2021

Thiago Emanuel Rodrigues Novaes
ORCID: https://orcid.org/0000-0002-3214-3220
Universidade Federal da Fronteira Sul, Brasil
E-mail: thiago.emanuel_rodri@ @otmail.com
Ana Selia Rodrigues Novaes
ORCID: https://orcid.org/0000-0002-9193-580X
E-mail: anaseliarn@ @ hotmail.com
Lissandra Glusczak
Instituto Federal de Educação, Ciência e Tecnologia do Sertã Pernambuano, Brasil
ORCID: https://orcid.org/0000-0002-7723-8901
Universidade Federal da Fronteira Sul, Brasil
E-mail: lissandra.glusczak@uffs.edu.br
Lucianne Braga Oliveira Vilarinho
ORCID: https://orcid.org/0000-0002-9178-8061
Universidade Federal da Fronteira Sul, Brasil
E-mail: lucianne.braga@ uffs.edu.br

\begin{abstract}
Resumo
A Caatinga, bioma exclusivamente brasileiro, abriga grande biodiversidade e potenciais medicinais em algumas espécies vegetais benéficas para a saúde humana, como a Cnidoscolus quercifolius, planta nativa desse bioma, popularmente conhecida como favela ou faveleira, que é bastante usada na medicina popular do sertão nordestino do Brasil. Nessa perspectiva, o presente estudo objetivou discorrer acerca dos usos medicinais da faveleira $(C$. quercifolius) na medicina popular nordestina. Trata-se de um estudo transversal descritivo, do tipo revisão de literatura, desenvolvido a partir de análises em trabalhos científicos publicados no período compreendido entre $2001 \mathrm{e}$ 2020, disponibilizados na base de dados Google Acadêmico, utilizando os descritores: "faveleira medicinal", "faveleira medicina popular" "Cnidoscolus quercifolius" e "Cnidoscolus quercifolius medicina popular". A partir dos dados coletados, observou-se que a faveleira (C. quercifolius) é usada amplamente na medicina popular nordestina nos tratamentos de processos inflamatórios, dores, infecções, cicatrizações e outros, além de possuir atividades antitumorais. Diante disso, é notório como a faveleira (C. quercifolius), possui amplas propriedades medicinais, sendo utilizada ao decorrer de várias gerações em tratamentos para a recuperação da saúde humana.
\end{abstract}

Palavras-chave: Saúde pública; Plantas medicinais; Medicina tradicional; Cnidoscolus quercifolius.

\begin{abstract}
The Caatinga, exclusively brazilian biome, harbors great biodiversity and medicinal potentials in some plant species beneficial to human health, such as Cnidoscolus quercifolius, a native plant of this biome, popularly known as favela or faveleira, which is widely used in folk medicine in the northeastern hinterland. of Brazil. In this perspective, the present study aimed to discuss the medicinal uses of the faveleira (C. quercifolius) in northeastern popular medicine. This is a descriptive cross-sectional study, such as a literature review, developed from analyzes in scientific works published between 2001 and 2020, made available in the Google Scholar database, using the descriptors: "faveleira medicinal", "faveleira medicina popular" "Cnidoscolus quercifolius" e "Cnidoscolus quercifolius medicina popular". From the data collected, it was observed that the faveleira (C. quercifolius) is widely used in Northeastern folk medicine in the treatment of inflammatory processes, pain, infections, scarring and others, besides having antitumor activities. Therefore, it is notorious as the faveleira (C. quercifolius), has wide medicinal properties, being used over several generations in treatments for the recovery of human health.
\end{abstract}

Keywords: Public health; Medicinal plants; Traditional medicine; Cnidoscolus quercifolius. 


\begin{abstract}
Resumen
La Caatinga, bioma exclusivamente brasileño, alberga una gran biodiversidad y potencialidades medicinales en algunas especies vegetales beneficiosas para la salud humana, como la Cnidoscolus quercifolius, una planta originaria de este bioma, conocida popularmente como favela o faveleira, muy utilizada en la medicina popular del interior del nordeste de Brasil. En esta perspectiva, el presente estudio tiene como objetivo discutir los usos medicinales de la faveleira (C. quercifolius) en la medicina popular del nordeste. Se trata de un estudio descriptivo transversal, del tipo revisión bibliográfica, desarrollado a partir de análisis en trabajos científicos publicados en el período comprendido entre 2001 y 2020, puestos a disposición en la base de datos de Google Académico, utilizando los descriptores: "faveleira medicinal", "faveleira medicina popular" "Cnidoscolus quercifolius" e "Cnidoscolus quercifolius medicina popular". A partir de los datos recogidos, se observó que la faveleira se utiliza ampliamente en la medicina popular del nordeste en el tratamiento de procesos inflamatorios, dolor, infección, cicatrización y otros, además de tener actividades antitumorales. En vista de ello, se conoce como la faveleira ( $C$. quercifolius), tiene amplias propiedades medicinales, siendo utilizada durante varias generaciones en tratamientos para la recuperación de la salud humana.

Palabras clave: Salud pública; Plantas medicinales; Medicina tradicional; Cnidoscolus quercifolius.
\end{abstract}

\title{
1. Introdução
}

A Caatinga, bioma exclusivamente brasileiro, abriga grande biodiversidade, variedade e riqueza de espécies botânicas que possuem diversas aplicabilidades, sejam elas fitoterápicas, uso forrageiro, na culinária e outras. Nessa perspectiva, o bioma abrange todos os nove estados da região Nordeste e parte da região Sudeste do Brasil. Entretanto, de acordo com Giulietti, et al. (2004),

Dentre os biomas brasileiros, a Caatinga é, provavelmente, o mais desvalorizado e mal conhecido botanicamente. Esta situação é decorrente de uma crença injustificada, e que não deve ser mais aceita, de que a Caatinga é o resultado da modificação de uma outra formação vegetal, estando associada a uma diversidade muito baixa de plantas, sem espécies endêmicas e altamente modificada pelas ações antrópicas.

As espécies vegetais nativas da Caatinga ainda são pouco exploradas em estudos experimentais para comprovação de atividades que corroborem sua eficácia no combate a doenças que acometem o sertanejo, mas que têm usos populares tradicionais passados e recomendados de geração em geração através, principalmente, da oralidade.

Dentre as inúmeras espécies nativas do bioma, podemos destacar a Cnidoscolus quercifolius, conhecida popularmente como faveleira, que é amplamente utilizada na cultura popular nordestina para tratamento de enfermidades, em decorrência de suas propriedades medicinais que potencializam esses usos. A faveleira também é conhecida por outros nomes, tais como favela, faveleiro, mandioca-brava ou favela-de-cachorro, dependendo da região onde é encontrada (Ferreira, 1986; Henrique, 2017).

\subsection{Cultura Popular Nordestina - Etnofarmacoterapia com o bioma Caatinga}

O conhecimento sertanejo acerca dos usos terapêuticos das diversas plantas do bioma Caatinga é, em geral, muito amplo. De acordo com Morais, et al. (2016), “o uso de medicamentos obtidos a partir de fontes vegetais é antigo, mas tem crescido nos últimos anos a procura por produtos naturais com atividades farmacológicas que trazem benefícios para a humanidade". Sabe-se que

No Nordeste do Brasil, apesar da grande influência dos meios de comunicação e do número crescente de farmácias na região, o uso de plantas medicinais ainda é frequente, tanto no meio rural e urbano, sendo comum principalmente neste último, a presença de raizeiros em pontos estratégicos de algumas cidades (Mosca \& Loiola, 2009).

A utilização das plantas do bioma Caatinga pelos nordestinos também está associada a questões socioculturais e socioeconômicas, principalmente nas áreas em que os serviços de saúde não conseguem abranger toda a população de maneira eficiente; desta forma, a população tem na medicina tradicional uma alternativa para suprir suas necessidades na promoção de 
saúde (Lisboa, et al., 2006; Oliveira, 2007).

\subsection{Importância da etnobotânica na Saúde Pública}

Muitos profissionais de saúde, na contemporaneidade, precisam lidar com questões socioculturais quando prestam atendimento ao paciente e a seus familiares na unidade de saúde em que atuam. Para efetivar o planejamento adequado de assistência, também devem levar em consideração os fatores culturais desses indivíduos e as diversas possibilidades terapêuticas, podendo fazer uso dos recursos fitoterápicos existentes e melhorar o nível de saúde da população (Araújo, 2002; Bruning; Mosegui \& Vianna, 2012).

Para isso, se faz necessário um conhecimento por parte dos profissionais de saúde que atuam diretamente com os pacientes nas UBS, em relação às propriedades terapêuticas das plantas que são usadas por essa população. Conhecimentos técnicos, que vão desde o preparo para fins terapêuticos, indicações, cuidados e dosagem, e conhecimentos sobre a percepção quanto à relação saúde-doença são imprescindíveis (Bruning; Mosegui \& Vianna, 2012).

Rodrigues, et al. (2020) ressaltam a importância de projetos que fazem uso das plantas medicinais nas unidades de saúde para o combate de muitas enfermidades, possibilitando a devida orientação para o emprego destas nos programas de Atenção Primária à Saúde (APS) e para o êxito do programa de pesquisa nesta área, além de prestar assistência farmacêutica fitoterápica de base científica.

A aplicação de estudos etnobotânicos na Saúde Pública se demonstra eficaz na valorização da cultura local de uma comunidade. Nesse sentido, representa possibilidade de compartilhar conhecimentos intergeracionais da medicina tradicional para contribuir na promoção de saúde, prevenindo, tratando e curando enfermidades.

Desse modo, empreendeu-se o seguinte questionamento para a construção do estudo: quais são os potenciais medicinais da faveleira (Cnidoscolus quercifolius) para a saúde humana no Sertão Nordestino?

Nessa perspectiva, o presente trabalho tem como objetivo discorrer acerca dos usos etnofarmacológicos da faveleira (Cnidoscolus quercifolius), planta nativa do bioma Caatinga, na cultura popular nordestina do Brasil para a promoção da saúde pública.

\section{Metodologia}

O presente trabalho se trata de um estudo transversal descritivo exploratório do tipo revisão de literatura, que se baseou em estudos acadêmicos e científicos produzidos em relação à etnofarmacologia, à cultura popular nordestina, aos usos múltiplos da Cnidoscolus quercifolius (faveleira), em especial dos usos na medicina popular, e às propriedades medicinais da espécie em questão. Para isso, pesquisou-se artigos científicos e trabalhos de conclusão de mestrado e doutorado, estudos de caráter exploratório e experimental, que abordassem as temáticas mencionadas.

A pesquisa pelos trabalhos se deu na base de dados Google Acadêmico e se utilizou dos seguintes descritores: "faveleira medicinal", "faveleira medicina popular" "Cnidoscolus quercifolius" e "Cnidoscolus quercifolius medicina popular".

Os termos da busca resultaram em centenas de estudos, mas foram avaliados os que apresentavam maior relevância por serem mais citados de acordo com a base de dados. Elegeu-se trabalhos científicos publicados em língua portuguesa e inglesa, que possuíam o período de publicação compreendido de 2001 a 2020. Os dados desta pesquisa foram coletados em julho de 2020. Nesse sentido, foram excluídos desta revisão os estudos que não se adequavam ao objetivo proposto. A condução deste artigo se deu de julho de 2020 a fevereiro de 2021. 
Os dados encontrados na pesquisa foram organizados em tabelas e discutidos na estrutura textual desta revisão. Além disso, utilizou-se de registros fotográficos para melhor identificação da faveleira (Cnidoscolus quercifolius).

Nesse sentido, esta seção está fundamentada na metodologia desenvolvida por Novaes \& Novaes (2021), ao estudarem os potenciais medicinais do cajueiro (Anacardium occidentale), outra espécie vegetal nativa do bioma Caatinga.

\section{Resultados e Discussão}

A Tabela 1 dispõe dos estudos eleitos (14 trabalhos) para compor esta breve revisão de literatura acerca dos potenciais medicinais da faveleira (Cnidoscolus quercifolius).

Tabela 1. Estudos eleitos para a presente revisão.

\begin{tabular}{|c|c|c|}
\hline Título & $\begin{array}{l}\text { Autoria e } \\
\text { ano }\end{array}$ & Local de publicação \\
\hline $\begin{array}{l}\text { A faveleira (Cnidoscolus quercifolius) como fonte alternativa na alimentação humana } \\
\text { e animal no Semi-Árido Paraibano }\end{array}$ & $\begin{array}{l}\text { Nóbrega } \\
(2001)\end{array}$ & $\begin{array}{c}\text { Universidade Federal } \\
\text { da Paraíba } \\
\text { (dissertação mestrado) }\end{array}$ \\
\hline $\begin{array}{l}\text { Estudo microbiológico da ação de extratos vegetais hidroalcoólicos sobre } \\
\text { microorganismos bucais }\end{array}$ & $\begin{array}{c}\text { Lacerda } \\
(2012)\end{array}$ & $\begin{array}{c}\text { Universidade Estadual } \\
\text { da Paraíba } \\
\text { (monografia) }\end{array}$ \\
\hline $\begin{array}{l}\text { Phytochemical screening and anti-inflammatory activity of Cnidoscolus quercifolius } \\
\text { (Euphorbiaceae) in mice }\end{array}$ & $\begin{array}{l}\text { Gomes, et al. } \\
\quad(2014 \mathrm{a})\end{array}$ & $\begin{array}{l}\text { Pharmacognosy } \\
\text { Research }\end{array}$ \\
\hline $\begin{array}{l}\text { Antinociceptive activity of the ethanolic extract from barks and leaves of Cnidoscolus } \\
\text { quercifolius (Euphorbiaceae) in mice }\end{array}$ & $\begin{array}{l}\text { Gomes, et al. } \\
(2014 \mathrm{~b})\end{array}$ & $\begin{array}{c}\text { Journal of Young } \\
\text { Pharmacists }\end{array}$ \\
\hline $\begin{array}{c}\text { Percepção da população das áreas com a presença da faveleira sobre os usos do } \\
\text { vegetal }\end{array}$ & $\begin{array}{c}\text { Medeiros \& } \\
\text { Aloufa (2016) }\end{array}$ & $\begin{array}{l}\text { Revista da Casa da } \\
\text { Geografia de Sobral }\end{array}$ \\
\hline $\begin{array}{c}\text { Chemical composition of faveleira (Cnidoscolus phyllacanthus) seeds collected in } \\
\text { different seasons. Revista Ceres, 65(3), 286-290. }\end{array}$ & $\begin{array}{l}\text { Medeiros et } \\
\text { al. (2018) }\end{array}$ & Revista Ceres \\
\hline $\begin{array}{c}\text { Prospecção fitoquímica e avaliação do potencial antioxidante de Cnidoscolus } \\
\text { phyllacanthus (müll. Arg.) Pax \& k. hoffm. Oriundo de Apodi-RN }\end{array}$ & $\begin{array}{l}\text { Morais, et al. } \\
\quad(2016)\end{array}$ & $\begin{array}{l}\text { Revista Brasileira de } \\
\text { Plantas Medicinais }\end{array}$ \\
\hline Constituintes químicos e atividade citotóxica de Cnidoscolus phyllacanthus & $\begin{array}{l}\text { Paula, et al. } \\
\text { (2016) }\end{array}$ & $\begin{array}{l}\text { Revista Virtual de } \\
\text { Química }\end{array}$ \\
\hline $\begin{array}{c}\text { Bioactive properties of faveleira (Cnidoscolus quercifolius) seeds, oil and press cake } \\
\text { obtained during oilseed processing }\end{array}$ & $\begin{array}{l}\text { Ribeiro, et al. } \\
\text { (2017) }\end{array}$ & PloS One \\
\hline $\begin{array}{l}\text { Chemical composition, antioxidant activity and thermal analysis of oil extracted from } \\
\text { favela (Cnidoscolus quercifolius) seeds }\end{array}$ & $\begin{array}{l}\text { Santos, et al. } \\
(2017)\end{array}$ & $\begin{array}{l}\text { Industrial Crops and } \\
\text { Products }\end{array}$ \\
\hline $\begin{array}{l}\text { Influência do método extrativo no teor de flavonoides de Cnidoscolus quercifolius } \\
\text { POHL (Euphorbiaceae) e atividade antioxidante }\end{array}$ & $\begin{array}{l}\text { Torres, et al. } \\
\text { (2018) }\end{array}$ & Química Nova \\
\hline $\begin{array}{l}\text { Plantas medicinais da caatinga do Nordeste brasileiro: etnofarmacopeia do Professor } \\
\text { Francisco José de Abreu Matos }\end{array}$ & $\begin{array}{l}\text { Magalhães } \\
(2019)\end{array}$ & $\begin{array}{c}\text { Universidade Federal } \\
\text { do Ceará (tese } \\
\text { doutorado) }\end{array}$ \\
\hline $\begin{array}{c}\text { Ethnobotanic, phytochemical uses and ethnopharmacological profile of genus } \\
\text { Cnidoscolus spp. (Euphorbiaceae): A comprehensive overview }\end{array}$ & $\begin{array}{l}\text { Moura, et al. } \\
(2019)\end{array}$ & $\begin{array}{c}\text { Biomedicine \& } \\
\text { Pharmacotherapy }\end{array}$ \\
\hline $\begin{array}{c}\text { Phenolic profiles of faveleira (Cnidoscolus quercifolius Pohl) seed and press cake } \\
\text { extracts: potential for a new trend in functional food }\end{array}$ & $\begin{array}{l}\text { Ribeiro, et al. } \\
\text { (2020) }\end{array}$ & $\begin{array}{l}\text { Brazilian Journal of } \\
\text { Food Technology }\end{array}$ \\
\hline
\end{tabular}

Fonte: Autores (2021).

A Tabela 2 apresenta os principais achados nos estudos, resumidamente. 
Tabela 2. Descrição de achados nos estudos.

\section{Autoria e ano}

Nóbrega (2001), Lacerda (2012), Medeiros \& Aloufa (2016), Morais, et al. (2016), Paula, et al. (2016), Torres, et al. (2018)

Gomes, et al. (2014a), Gomes, et al. (2014b), Morais, et al. (2016), Torres, et al. (2018), Magalhães (2019)

Ribeiro, et al. (2017), Medeiros, et al. (2018), Santos, et al. (2017), Moura, et al. (2019), Ribeiro, et al. (2020)

Fonte: Autores (2021).

Muitas famílias de plantas e ervas da Caatinga usadas no tratamento tradicional de doenças têm valor medicinal, proporcionando a prevenção, o alívio ou a cura de várias enfermidades humanas (Bitu, et al., 2015).

A utilização da faveleira (Figura 1) em procedimentos terapêuticos populares, na cultura nordestina, é relatada em vários estudos por apresentar propriedades analgésicas, antibióticas, anti-inflamatórias, cicatrizantes e diuréticas (Nóbrega, 2001). Segundo Lacerda (2012), a atividade antimicrobiana pode ser confirmada em razão da faveleira ter sido evidenciada como inibidora de bactérias patogênicas.

Figura 1. Cnidoscolus quercifolius (faveleira) em primeiro plano.

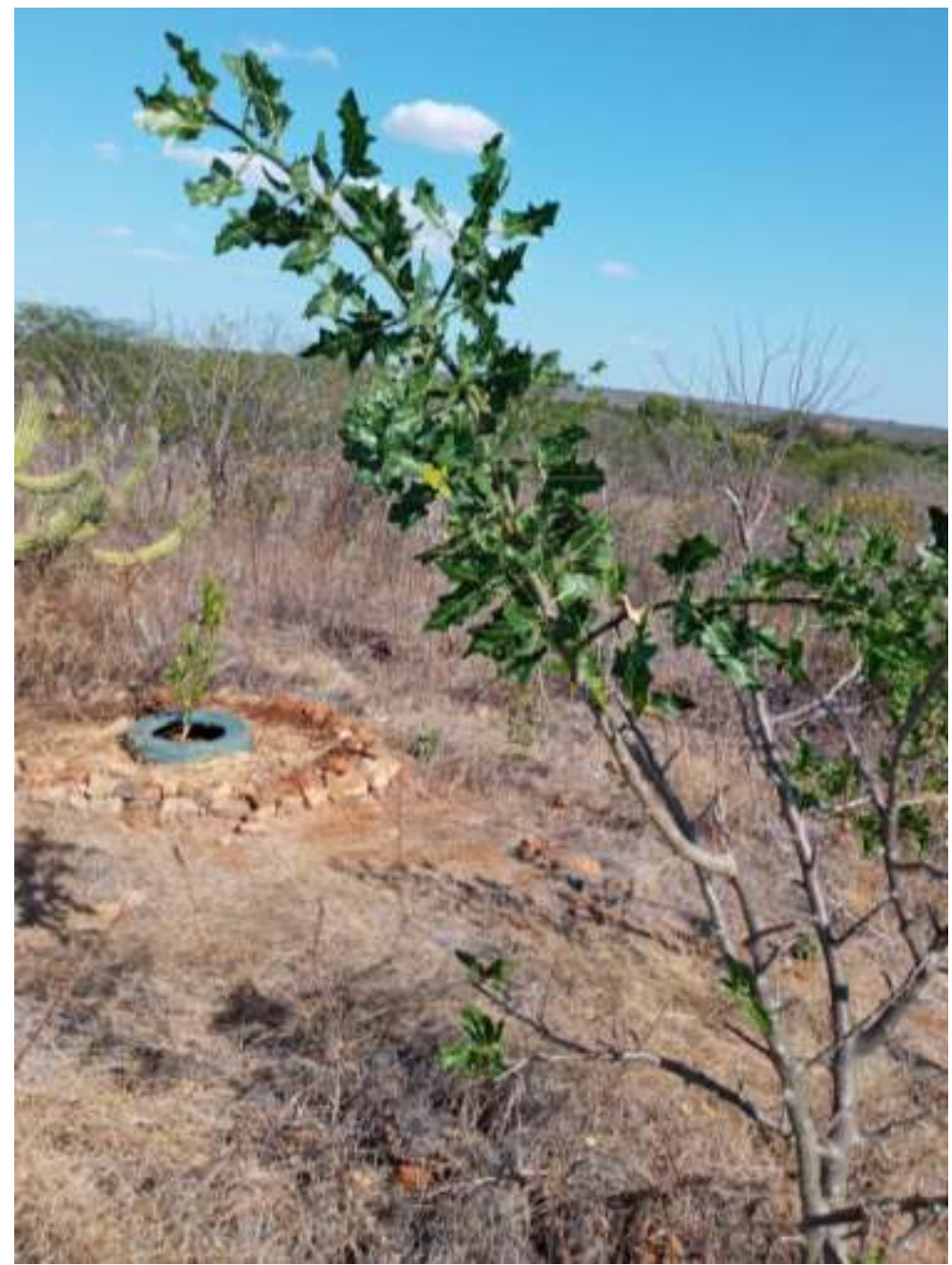

Fonte: Autores (2021). 
Os extratos de Cnidoscolus quercifolius demonstraram que, "dentre as diversas propriedades apresentadas pelos metabólitos secundários biossintetizados pelos vegetais, a atividade antioxidante, exibida por muitas plantas de uso comum na medicina tradicional apresenta destaque" (Morais et al., 2016). É valido destacar que as características químicas dos metabólitos secundários dependem de fatores que estão relacionados ao ambiente em que se encontram (Kutchan, 2001; Gobbo-Neto \& Lopes, 2007), dessa maneira, o bioma Caatinga condiciona a qualidade da síntese dos metabólitos da faveleira.

Nesse sentido, Gomes, et al. (2014a) e Morais, et al. (2016) relataram nas suas pesquisas a presença de cumarinas, fenóis, flavonoides do tipo flavonas, flavonóis, mononoterpenos, diterpenos, saponinas, triterpenóides pentacíclicos livres e xantonas, corroborando atividades antioxidantes e medicinais nos extratos de faveleira.

Outros estudos sugerem que extratos etanólicos obtidos das folhas e cascas do caule da C. quercifolius (faveleira) também apresentam atividade antinociceptiva (Gomes, et al., 2014b).

De acordo com Torres, et al. (2018),

[...] as atividades farmacológicas verificadas para C. quercifolius são normalmente atribuídas a classes de metabólitos secundários distintas, o que torna necessário o desenvolvimento de métodos extrativos que favoreçam a obtenção de compostos de interesse para determinadas propriedades terapêuticas.

Segundo Magalhães (2019), a flavonoide rutina da C. quercifolius (faveleira) pode ser associada em conjunto a outras espécies vegetais, como a Eremanthus erythropappus (candeia), no tratamento de insuficiência venosa.

O estudo de Medeiros e Aloufa (2016) indicou relatos de cura de ferimentos e na obstrução de sangramentos por ocasião de acidentes e no combate a verminoses, com uso do caule da C. quercifolius.

Ademais, Paula, et al. (2016) e Torres, et al. (2018) realizaram estudos que verificaram a presença de marcadores quimiotaxonômicos em extratos das cascas do caule da planta, que apresentam atividade citotóxica contra células tumorais humanas, o que atribui atividade antitumoral.

Além de possuir propriedades fitoterápicas, a faveleira possui usos múltiplos no Sertão Nordestino. Destaca-se sobre outras espécies do bioma Caatinga em decorrência de sua alta disseminação e completa adaptação às condições adversas (Pordeus Neto, et al., 2009; Ribeiro, 2016).

As sementes da faveleira (Figura 2) são consumidas por humanos há muito tempo, mas sua aplicação industrial e seu potencial tecnológico ainda não foram totalmente explorados. Sabe-se que a semente da faveleira por ser oleaginosa,possui alto valor nutricional, pois as proteínas e os lipídios são seus principais componentes. Portanto, a semente pode ser ingerida in natura, ou seja, na sua forma natural, ou adicionada a bolos e biscoitos, além de ser uma fonte potencial de óleo comestível (Ribeiro, et al., 2017; Medeiros, et al., 2018; Santos, et al., 2017; Moura, et al., 2019; Ribeiro, et al., 2020). 
Figura 2. Sementes de Cnidoscolus quercifolius (faveleira) in natura, que podem ser usadas na alimentação humana.

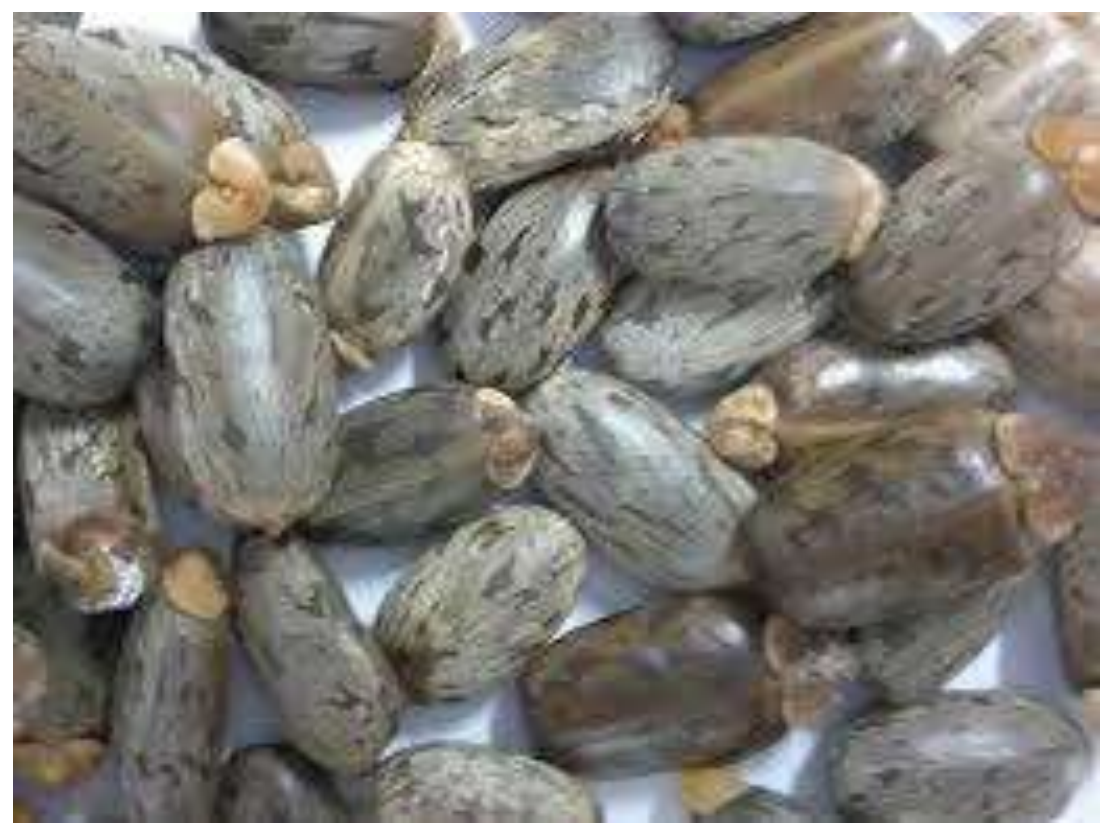

Fonte: Ribeiro (2016).

\section{Considerações Finais}

Diante desses achados, é notório como a faveleira (C. quercifolius) possui amplas propriedades medicinais relevantes, sendo utilizada como tratamento terapêutico pela cultura popular nordestina no decorrer de várias gerações. São múltiplos os usos na saúde para a espécie vegetal nativa em questão, destacando-se nos tratamentos de processos inflamatórios, dores, infecções e cicatrizações, além de possuir atividades antioxidantes e antitumorais.

Outrossim, ressalta-se a necessidade de mais estudos científicos e publicações sobre as propriedades e usos seguros da Cnidoscolus quercifolius na medicina popular. Além de ampliar a divulgação de suas aplicações na cultura nordestina, visando efetivar e melhorar a promoção de saúde para a população.

\section{Referências}

Araújo, M. A. M. (2002). Das ervas medicinais à fitoterapia. Ateliê Editorial.

Bitu, V. D. C. N., Bitu, V. D. C. N., Matias, E. F. F., de Lima, W. P., da Costa Portelo, A., Coutinho, H. D. M., \& de Menezes, I. R. A. (2015). Ethnopharmacological study of plants sold for therapeutic purposes in public markets in Northeast Brazil. Journal of Ethnopharmacology, 172, $265-272$.

Bruning, M. C. R., Mosegui, G. B. G., \& Vianna, C. M. D. M. (2012). A utilização da fitoterapia e de plantas medicinais em unidades básicas de saúde nos municípios de Cascavel e Foz do Iguaçu-Paraná: a visão dos profissionais de saúde. Ciência \& saúde coletiva, 17, 2675-2685.

Ferreira, A. B. D. H. (1986). Novo dicionário da língua portuguesa. In Novo dicionário da língua portuguesa (pp. 1838-1838).

Giulietti, A. M., Bocage Neta, A. L., Castro, A. A. J. F., Gamarra-Rojas, C. F. L., Sampaio, E. V. S. B., Virgínio, J. F., \& Harley, R. M. (2004). Diagnóstico da vegetação nativa do bioma Caatinga. Biodiversidade da Caatinga: áreas e ações prioritárias para a conservação.

Gobbo-Neto, L., \& Lopes, N. P. (2007). Plantas medicinais: fatores de influência no conteúdo de metabólitos secundários. Química nova, 30(2), $374-381$.

Gomes, L. M. A., Andrade, T. M., Silva, J. C., de Lima, J. T., Quintans-Junior, L. J., \& Almeida, J. R. G. S. (2014a). Phytochemical screening and antiinflammatory activity of Cnidoscolus quercifolius (Euphorbiaceae) in mice. Pharmacognosy Research, 6(4), 345.

Gomes, L. M. A., Lima-Saraiva, S. R. G., Andrade, T. M. D., Silva, J. C., Diniz, T. C., Barreto, V. N. S., ... \& Almeida, J. R. G. S. (2014b). Antinociceptive activity of the ethanolic extract from barks and leaves of Cnidoscolus quercifolius (Euphorbiaceae) in mice. Journal of Young Pharmacists, 6(2), 64.

Kutchan, T. M. (2001). Ecological arsenal and developmental dispatcher. The paradigm of secondary metabolism. Plant physiology, 125(1), 58-60.

Lacerda, S. R. L. (2012). Estudo microbiológico da ação de extratos vegetais hidroalcoólicos sobre microorganismos bucais [monografia]. Campina Grande: Universidade Estadual da Paraíba. 
Lisboa, M. S., Ferreira, S. M., \& Silva, M. S. (2006). Uso de plantas medicinais para tratar úlceras e gastrites pela comunidade do Povoado Vila Capim, município de Arapiraca-AL, Nordeste do Brasil. Sitientibus Série Ciências Biológicas, 6, 13-20.

Magalhães, K. D. N. (2019). Plantas medicinais da caatinga do Nordeste brasileiro: etnofarmacopeia do Professor Francisco José De Abreu Matos. $220 \mathrm{f}$. Tese (Doutorado em Desenvolvimento e Inovação Tecnológica em Medicamentos) - Faculdade de Farmácia, Odontologia e Enfermagem, Universidade Federal do Ceará, Fortaleza.

Medeiros, J. A., \& Aloufa, M. A. I. (2016). Percepção da população das áreas com a presença da faveleira sobre os usos do vegetal. Revista da Casa da Geografia de Sobral, 18(1), 2.

Medeiros, J. M. S. D., Ribeiro, P. P. C., Freitas, E. P. S., Santos, J. A. B. D., \& Damasceno, K. S. F. D. S. C. (2018). Chemical composition of faveleira (Cnidoscolus phyllacanthus) seeds collected in different seasons. Revista Ceres, 65(3), 286-290.

Morais, N. R. L., Oliveira Neto, F. B., Melo, A. R., Bertini, L. M., Silva, F. F. M., \& Alves, L. A. (2016). Prospecção fitoquímica e avaliação do potencial antioxidante de Cnidoscolus phyllacanthus (müll. Arg.) Pax \& k. hoffm. Oriundo de apodi-RN. Revista Brasileira de plantas medicinais, 18(1), 180-185

Mosca, V. P., \& Loiola, M. I. B. (2009). Uso popular de plantas medicinais no Rio Grande do Norte, nordeste do Brasil. Revista Caatinga, 22(4), $225-234$.

Moura, L. F. W. G., da Silva Neto, J. X., Lopes, T. D. P., Benjamin, S. R., Brito, F. C. R., Magalhães, F. E. A., \& Guedes, M. I. F. (2019). Ethnobotanic, phytochemical uses and ethnopharmacological profile of genus Cnidoscolus spp.(Euphorbiaceae): A comprehensive overview. Biomedicine \& Pharmacotherapy, 109, 1670-1679.

Nobrega, S. B. P. (2001). A faveleira (Cnidoscolus quercifolius) como fonte alternativa na alimentação humana e animal, no semi-árido paraibano. 145p. Dissertação (Mestrado em Desenvolvimento e Meio Ambiente) - Universidade Federal da Paraíba, João Pessoa.

Novaes, T. E. R., \& Novaes, A. S. R. (2021). Análise dos potenciais medicinais do cajueiro (Anacardium occidentale Linn): uma breve revisão. Research, Society and Development, 10(1), e41810111838-e41810111838.

Oliveira, G. L. (2007). Etnobotânica nordestina: plantas medicinais da comunidade Muribeca (Jaboatão dos Guararapes PE, Brasil) (Master's thesis, Universidade Federal de Pernambuco).

Paula, A. C., Melo, K. M., da Silva, A. M., Ferreira, D. A., Monte, F. J., Santiago, G. M., ... \& da Silva, T. G. (2016). Constituintes químicos e atividade citotóxica de Cnidoscolus phyllacanthus. Revista Virtual de Química, 8(1), 231-241.

Pordeus Neto, J., Rodrigues, O. G., Lima, E. Q., Marinho, M. G. V., \& Dantas, J. P. (2009). Avaliação da ação clastogênica do óleo de Cnidoscolus phyllacanthus (mart.) pax. et k. hoffm em células medulares. Revista de Biologia e Farmácia, 3(1), 6-22.

Ribeiro, P. P. C. (2016). Caracterização físico-químicas do óleo da semente de faveleira (Cnidoscolus quercifolius) e avaliação das propriedades bioativas da semente, do óleo e da torta (Master's thesis, Brasil).

Ribeiro, P. P. C., Silva, D. M. D. L. E., Assis, C. F. D., Correia, R. T. P., \& Damasceno, K. S. F. D. S. C. (2017). Bioactive properties of faveleira (Cnidoscolus quercifolius) seeds, oil and press cake obtained during oilseed processing. PloS one, 12(8), e0183935.

Ribeiro, P. P. C., Sousa Júnior, F. C. D., Assis, C. F. D., Veras, B. O. D., Padilha, C. E. D. A., Stamford, T. C. M., \& Damasceno, K. S. F. D. S. C. (2020). Phenolic profiles of faveleira (Cnidoscolus quercifolius Pohl) seed and press cake extracts: potential for a new trend in functional food. Brazilian Journal of Food Technology, 23

Rodrigues, T. A, Neto, J. L., Carvalho, T. D. A. R., Barbosa, M. E., Guedes, J. C., \& de Carvalho, A. V. (2020). A valorização das plantas medicinais como alternativa à saúde: um estudo etnobotânico. Revista Ibero-Americana de Ciências Ambientais, 11(1), 411-428.

Santos, K. A., Aragão Filho, O. P., Aguiar, C. M., Milinsk, M. C., Sampaio, S. C., Palú, F., \& da Silva, E. A. (2017). Chemical composition, antioxidant activity and thermal analysis of oil extracted from favela (Cnidoscolus quercifolius) seeds. Industrial Crops and Products, 97, 368-373.

Torres, D. D. S., Pereira, E. C., Sampaio, P. A., de Souza, N. A., Ferraz, C. A., Oliveira, A. P. D., \& Rolim, L. A. (2018). Influência do método extrativo no teor de flavonoides de Cnidoscolus quercifolius POHL (Euphorbiaceae) e atividade antioxidante. Química Nova, 41(7), 743-747. 